$\mathrm{J}$ o u r n a l of

Mathematics

and Applications

JMA No 36, pp 95-111 (2013)

\title{
On a study of double gai sequence space
}

\author{
N. Subramanian, U. K. Misra
}

Submitted by: Jan Stankiewicz

ABstract: Let $\chi^{2}$ denote the space of all prime sense double gai sequences and $\Lambda^{2}$ the space of all prime sense double analytic sequences.

This paper is devoted to the general properties of $\chi^{2}$.

AMS Subject Classification: 40A05,40C05,40D05

Keywords and Phrases: gai sequence, analytic sequence, double sequence, dual, monotone metric.

\section{Introduction}

Throughout $w, \chi$ and $\Lambda$ denote the classes of all, gai and analytic scalar valued single sequences, respectively. We write $w^{2}$ for the set of all complex sequences $\left(x_{m n}\right)$, where $m, n \in \mathbb{N}$, the set of positive integers. Then, $w^{2}$ is a linear space under the coordinate wise addition and scalar multiplication.

Some initial works on double sequence spaces is found in Bromwich[4]. Later on, they were investigated by Hardy[8], Moricz[12], Moricz and Rhoades[13], Basarir and Solankan[2], Tripathy[20], Colak and Turkmenoglu[6], Turkmenoglu[22], and many others.

Let us define the following sets of double sequences:

$$
\begin{aligned}
\mathcal{M}_{u}(t) & :=\left\{\left(x_{m n}\right) \in w^{2}: \sup _{m, n \in N}\left|x_{m n}\right|^{t_{m n}}<\infty\right\}, \\
\mathcal{C}_{p}(t) & :=\left\{\left(x_{m n}\right) \in w^{2}: p-\lim _{m, n \rightarrow \infty}\left|x_{m n}-l\right|^{t_{m n}}=1 \text { for somel } \in \mathbb{C}\right\}, \\
\mathcal{C}_{0 p}(t) & :=\left\{\left(x_{m n}\right) \in w^{2}: p-\lim _{m, n \rightarrow \infty}\left|x_{m n}\right|^{t_{m n}}=1\right\}, \\
\mathcal{L}_{u}(t) & :=\left\{\left(x_{m n}\right) \in w^{2}: \sum_{m=1}^{\infty} \sum_{n=1}^{\infty}\left|x_{m n}\right|^{t_{m n}}<\infty\right\}, \\
\mathcal{C}_{b p}(t) & :=\mathcal{C}_{p}(t)
\end{aligned}
$$

COPYRIGHT (c) by Publishing Department Rzeszów University of Technology P.O. Box 85, 35-959 Rzeszów, Poland 
where $t=\left(t_{m n}\right)$ is the sequence of strictly positive reals $t_{m n}$ for all $m, n \in \mathbb{N}$ and $p-\lim _{m, n \rightarrow \infty}$ denotes the limit in the Pringsheim's sense. In the case $t_{m n}=1$ for all $m, n \in \mathbb{N} ; \mathcal{M}_{u}(t), \mathcal{C}_{p}(t), \mathcal{C}_{0 p}(t), \mathcal{L}_{u}(t), \mathcal{C}_{b p}(t)$ and $\mathcal{C}_{0 b p}(t)$ reduce to the sets $\mathcal{M}_{u}, \mathcal{C}_{p}, \mathcal{C}_{0 p}, \mathcal{L}_{u}, \mathcal{C}_{b p}$ and $\mathcal{C}_{0 b p}$, respectively.

Now, we may summarize the knowledge given in some document related to the double sequence spaces. Gökhan and Colak $[27,28]$ have proved that $\mathcal{M}_{u}(t)$ and $\mathcal{C}_{p}(t), \mathcal{C}_{b p}(t)$ are complete paranormed spaces of double sequences and gave the $\alpha-, \beta-, \gamma-$ duals of the spaces $\mathcal{M}_{u}(t)$ and $\mathcal{C}_{b p}(t)$. Quite recently, in her PhD thesis, Zelter [29] has essentially studied both the theory of topological double sequence spaces and the theory of summability of double sequences. Mursaleen and Edely [30] have recently introduced the statistical convergence and Cauchy for double sequences and given the relation between statistical convergent and strongly Cesàro summable double sequences. Nextly, Mursaleen [31] and Mursaleen and Edely [32] have defined the almost strong regularity of matrices for double sequences and applied these matrices to establish a core theorem and introduced the $M$-core for double sequences and determined those four dimensional matrices transforming every bounded double sequences $x=\left(x_{j k}\right)$ into one whose core is a subset of the $M$-core of $x$. More recently, Altay and Basar [33] have defined the spaces $\mathcal{B S}, \mathcal{B S}(t), \mathcal{C} \mathcal{S}_{p}, \mathcal{C} \mathcal{S}_{b p}, \mathcal{C} \mathcal{S}_{r}$ and $\mathcal{B V}$ of double sequences consisting of all double series whose sequence of partial sums are in the spaces $\mathcal{M}_{u}, \mathcal{M}_{u}(t), \mathcal{C}_{p}, \mathcal{C}_{b p}, \mathcal{C}_{r}$ and $\mathcal{L}_{u}$, respectively, and also examined some properties of those sequence spaces and determined the $\alpha$-duals of the spaces $\mathcal{B S}, \mathcal{B V}, \mathcal{C S} \mathcal{S}_{b p}$ and the $\beta(\vartheta)$ - duals of the spaces $\mathcal{E} \mathcal{S}_{b p}$ and $\mathcal{C} \mathcal{S}_{r}$ of double series. Quite recently Basar and Sever [34] have introduced the Banach space $\mathcal{L}_{q}$ of double sequences corresponding to the well-known space $\ell_{q}$ of single sequences and examined some properties of the space $\mathcal{L}_{q}$. Quite recently Subramanian and Misra [35] have studied the space $\chi_{M}^{2}(p, q, u)$ of double sequences and gave some inclusion relations. We need the following inequality in the sequel of the paper. For $a, b, \geq 0$ and $0<p<1$, we have

$$
(a+b)^{p} \leq a^{p}+b^{p}
$$

The double series $\sum_{m, n=1}^{\infty} x_{m n}$ is called convergent if and only if the double sequence $\left(s_{m n}\right)$ is convergent, where $s_{m n}=\sum_{i, j=1}^{m, n} x_{i j}(m, n \in \mathbb{N})$ (see[1]).

A sequence $x=\left(x_{m n}\right)$ is said to be double analytic if $\sup _{m n}\left|x_{m n}\right|^{1 / m+n}<\infty$. The vector space of all double analytic sequences will be denoted by $\Lambda^{2}$. A sequence $x=\left(x_{m n}\right)$ is called double gai sequence if $\left((m+n) !\left|x_{m n}\right|\right)^{1 / m+n} \rightarrow 0$ as $m, n \rightarrow \infty$. The double gai sequences will be denoted by $\chi^{2}$. Let $\phi=\{$ allfinitesequences $\}$.

Consider a double sequence $x=\left(x_{i j}\right)$. The $(m, n)^{t h}$ section $x^{[m, n]}$ of the sequence is defined by $x^{[m, n]}=\sum_{i, j=0}^{m, n} x_{i j} \Im_{i j}$ for all $m, n \in \mathbb{N}$; where $\Im_{i j}$ denotes the double sequence whose only non zero term is a $\frac{1}{(i+j) !}$ in the $(i, j)^{t h}$ place for each $i, j \in \mathbb{N}$.

An FK-space(or a metric space) $X$ is said to have AK property if $\left(\Im_{m n}\right)$ is a Schauder basis for $X$. Or equivalently $x^{[m, n]} \rightarrow x$.

An FDK-space is a double sequence space endowed with a complete metrizable; locally convex topology under which the coordinate mappings $x=\left(x_{k}\right) \rightarrow\left(x_{m n}\right)(m, n \in$ 
$\mathbb{N}$ ) are also continuous.

If $X$ is a sequence space, we give the following definitions:

(i) $\quad X^{\prime}=$ the continuous dual of $X$;

(ii) $\quad X^{\alpha}=\left\{a=\left(a_{m n}\right): \sum_{m, n=1}^{\infty}\left|a_{m n} x_{m n}\right|<\infty\right.$, for each $\left.x \in X\right\}$;

(iii) $X^{\beta}=\left\{a=\left(a_{m n}\right): \sum_{m, n=1}^{\infty} a_{m n} x_{m n}\right.$ is convegent, foreach $\left.x \in X\right\}$;

(iv) $\quad X^{\gamma}=\left\{a=\left(a_{m n}\right): \sup _{m n} \geq 1\left|\sum_{m, n=1}^{M, N} a_{m n} x_{m n}\right|<\infty\right.$, foreach $\left.x \in X\right\}$;

(v) let $X$ beanF $K-$ space $\supset \phi$; then $X^{f}=\left\{f\left(\Im_{m n}\right): f \in X^{\prime}\right\}$;

(vi) quad $X^{\delta}=\left\{a=\left(a_{m n}\right): \sup _{m n}\left|a_{m n} x_{m n}\right|^{1 / m+n}<\infty\right.$, foreach $\left.x \in X\right\}$;

$X^{\alpha} \cdot X^{\beta}, X^{\gamma}$ are called $\alpha-($ or Köthe - Toeplitz)dual of $X, \beta-$ (or generalized Köthe-Toeplitz)dual of $X, \gamma-$ dual of $X, \delta-$ dual of $X$ respectively. $X^{\alpha}$ is defined by Gupta and Kamptan [24]. It is clear that $X^{\alpha} \subset X^{\beta}$ and $X^{\alpha} \subset X^{\gamma}$, but $X^{\alpha} \subset X^{\gamma}$ does not hold, since the sequence of partial sums of a double convergent series need not to be bounded.

The notion of difference sequence spaces (for single sequences) was introduced by Kizmaz [36] as follows

$$
Z(\Delta)=\left\{x=\left(x_{k}\right) \in w:\left(\Delta x_{k}\right) \in Z\right\}
$$

for $Z=c, c_{0}$ and $\ell_{\infty}$, where $\Delta x_{k}=x_{k}-x_{k+1}$ for all $k \in \mathbb{N}$. Here $w, c, c_{0}$ and $\ell_{\infty}$ denote the classes of all, convergent,null and bounded sclar valued single sequences respectively. The above spaces are Banach spaces normed by

$$
\|x\|=\left|x_{1}\right|+\sup _{k \geq 1}\left|\Delta x_{k}\right|
$$

Later on the notion was further investigated by many others. We now introduce the following difference double sequence spaces defined by

$$
Z(\Delta)=\left\{x=\left(x_{m n}\right) \in w^{2}:\left(\Delta x_{m n}\right) \in Z\right\}
$$

where $Z=\Lambda^{2}, \chi^{2}$ and $\Delta x_{m n}=\left(x_{m n}-x_{m n+1}\right)-\left(x_{m+1 n}-x_{m+1 n+1}\right)=x_{m n}-$ $x_{m n+1}-x_{m+1 n}+x_{m+1 n+1}$ for all $m, n \in \mathbb{N}$

\section{Definitions and Preliminaries}

A sequence $x=\left(x_{m n}\right)$ is said to be double analytic if

$$
\sup _{m n}\left|x_{m n}\right|^{1 / m+n}<\infty
$$

The vector space of all double analytic sequences is usually denoted by $\Lambda^{2}$. A sequence $x=\left(x_{m n}\right)$ is called double entire sequence if $\left|x_{m n}\right|^{1 / m+n} \rightarrow 0$ as $m, n \rightarrow \infty$. The vector space of double entire sequences is usually denoted by $\Gamma^{2}$. A sequence $x=\left(x_{m n}\right)$ is called double gai sequence if $\left((m+n) !\left|x_{m n}\right|\right)^{1 / m+n} \rightarrow 0$ as $m, n \rightarrow \infty$. The vector 
space of double gai sequences is usually denoted by $\chi^{2}$. The space $\chi^{2}$ is a metric space with the metric

$$
d(x, y)=\sup _{m, n}\left\{\left((m+n) !\left|x_{m n}-y_{m n}\right|\right)^{1 / m+n}: m, n: 1,2,3, \ldots\right\}
$$

for all $x=\left\{x_{m n}\right\}$ and $y=\left\{y_{m n}\right\}$ in $\chi^{2}$.

\section{Main Results}

Proposition $3.1 \chi^{2}$ has monotone metric.

Proof: We know that

$$
\begin{aligned}
d(x, y) & =\sup _{m n}\left\{\left((m+n) !\left|x_{m n}-y_{m n}\right|\right)^{1 / m+n}: m, n: 1,2,3, \ldots\right\} \\
d\left(x^{n}, y^{n}\right) & =\sup _{n, n}\left\{\left((2 n) !\left|x_{n n}-y_{n n}\right|\right)^{1 / 2 n}\right\}
\end{aligned}
$$

and

$$
d\left(x^{m}, y^{m}\right)=\sup _{m, m}\left\{\left((2 m) !\left|x_{m m}-y_{m m}\right|\right)^{1 / 2 m}\right\}
$$

Let $m>n$. Then

$$
\begin{aligned}
\sup _{m, m}\left\{\left((2 m) !\left|x_{m m}-y_{m m}\right|\right)^{1 / 2 m}\right\} & \geq \sup _{n, n}\left\{\left((2 n) !\left|x_{n n}-y_{n n}\right|\right)^{1 / 2 n}\right\} \\
d\left(x^{m}, y^{m}\right) & \geq d\left(x^{n}, y^{n}\right), \quad m>n
\end{aligned}
$$

Also $\left\{d\left(x^{n}, x^{n}\right): n=1,2,3, \ldots\right\}$ is monotonically increasing bounded by $d(x, y)$. For such a sequence

$$
\sup _{n, n}\left\{\left((2 n !)\left|x^{n n}-y^{n n}\right|\right)^{1 / 2 n}\right\}=n \stackrel{\lim }{\rightarrow} \infty d\left(x^{n}, y^{n}\right)=d(x, y)
$$

From(3) and (4) it follows that $d(x, y)=\sup _{m n}\left\{\left((m+n) !\left|x_{m n}-y_{m n}\right|\right)^{1 / m+n}\right\}$ is a monotone metric for $\chi^{2}$. This completes the proof.

Proposition 3.2 The dual space of $\chi^{2}$ is $\Lambda^{2}$.In other words $\left(\chi^{2}\right)^{*}=\Lambda^{2}$.

Proof: We recall that

$$
\Im_{m n}=\left(\begin{array}{ccccc}
0, & 0, & \ldots 0, & 0, & \ldots \\
0, & 0, & \ldots 0, & 0, & \ldots \\
\cdot & & & & \\
\cdot & & & & \\
\cdot & & & & \\
0, & 0, & \ldots \frac{1}{(m+n) !}, & 0, & \ldots \\
0, & 0, & \ldots 0, & 0, & \ldots
\end{array}\right)
$$


with $\frac{1}{(m+n) !}$ in the $(m, n)$ th position and zero's else where. With

$$
\begin{aligned}
& \begin{aligned}
x & =\Im_{m n},\left(\left|x_{m n}\right|\right)^{1 / m+n} \\
& =\left(\begin{array}{cccc}
0^{1 / 2}, . & \cdot & \cdot & 0^{1 / 1+n} \\
\cdot & & & \\
\cdot & & & \\
0^{1 / m+1}, & \left(\frac{1}{(m+n) !}\right)^{1 / m+n}, & \cdot & 0^{1 / m+n+1} \\
0^{1 / m+2}, & (m, n)^{t h} & & \\
\cdot & \cdot & 0^{1 / m+n+2}
\end{array}\right)
\end{aligned} \\
& =\left(\begin{array}{cccc}
0, . & \cdot & \cdot & 0 \\
\cdot & & & \\
\cdot & & & \\
\cdot & & 1 \\
0, & \left(\frac{1}{(m+n) !}\right)^{1 / m+n} & , & 0 \\
0, & (m, n)^{t h} & & 0
\end{array}\right)
\end{aligned}
$$

which is a double gai sequence. Hence $\Im_{m n} \in \chi^{2}$. We have $f(x)=\sum_{m, n=1}^{\infty} x_{m n} y_{m n}$. With $x \in \chi^{2}$ and $f \in\left(\chi^{2}\right)^{*}$ the dual space of $\chi^{2}$. Take $x=\left(x_{m n}\right)=\Im_{m n} \in \chi^{2}$.Then

$$
\left|y_{m n}\right| \leq\|f\| d\left(\Im_{m n}, 0\right)<\infty \quad \forall m, n
$$

Thus $\left(y_{m n}\right)$ is a bounded sequence and hence an double analytic sequence. In other words $y \in \Lambda^{2}$. Therefore $\left(\chi^{2}\right)^{*}=\Lambda^{2}$. This completes the proof.

Proposition $3.3 \chi^{2}$ is separable.

Proof:It is routine verification. Therefore omit the proof.

Proposition $3.4 \Lambda^{2}$ is not separable.

Proof:Since $\left|x_{m n}\right|^{1 / m+n} \rightarrow 0$ as $m, n \rightarrow \infty$,so it may so happen that first row or column may not be convergent, even may not be bounded. Let $S$ be the set that has double sequences such that the first row is built up of sequences of zeros and ones. Then $S$ will be uncountable. Consider open balls of radius $3^{-1}$ units. Then these open balls will not cover $\Lambda^{2}$.Hence $\Lambda^{2}$ is not separable. This completes the proof.

Proposition $3.5 \chi^{2}$ is not reflexive.

Proof: $\chi^{2}$ is separable by Proposition 3.3. But $\left(\chi^{2}\right)^{*}=\Lambda^{2}$, by Proposition 3.2. Since $\Lambda^{2}$ is not separable, by Proposition 3.4. Therefore $\chi^{2}$ is not reflexive. This completes the proof.

Proposition $3.6 \chi^{2}$ is not an inner product space as such not a Hilbert space. 
Proof: Let us take

$$
x=x_{m n}=\left(\begin{array}{ccccc}
1 / 2 !, & 1 / 3 !, & 0, & 0, & \ldots \\
0, & 0, & 0, & 0, & \ldots \\
\cdot & & & & \\
\cdot & & & & \\
\cdot & & & &
\end{array}\right)
$$

and

$$
\begin{aligned}
& y=y_{m n}=\left(\begin{array}{ccccc}
1 / 2 !, & -1 / 3 !, & 0, & 0, & \cdots \\
0, & 0, & 0, & 0, & \\
\vdots & \vdots & \vdots &
\end{array}\right) \\
& d(x, 0)=\sup \left(\begin{array}{ccc}
\left(2 !\left|x_{11}-0\right|\right)^{1 / 2}, & \left(3 !\left|x_{12}-0\right|\right)^{1 / 3}, & \ldots \\
\left(3 !\left|x_{21}-0\right|\right)^{1 / 3}, & \left(4 !\left|x_{22}-0\right|\right)^{1 / 4}, & \ldots \\
\vdots & \vdots & \vdots
\end{array}\right) \\
& =\sup \left(\begin{array}{ccc}
(2 !|1 / 2 !-0|)^{1 / 2}, & (3 !|1 / 3 !-0|)^{1 / 3}, & \ldots \\
0, & 0, & \cdots \\
\vdots & \vdots & \vdots
\end{array}\right) \\
& =\sup \left(\begin{array}{cccc}
(1)^{1 / 2}, & (1)^{1 / 3}, & 0, & \cdots \\
0, & 0, & 0, & \cdots \\
\vdots & \vdots & \vdots & \vdots
\end{array}\right) \\
& d(x, 0)=1 .
\end{aligned}
$$

Similarly $d(x, 0)=1$. Hence $d(x, 0)=d(y, 0)=1$

$$
\begin{aligned}
x+y & =\left(\begin{array}{ccccc}
1 / 2 !, & 1 / 3 !, & 0 & , 0 & \ldots \\
0, & 0, & 0, & 0, & \ldots \\
\vdots & \vdots & \vdots & \ddots & \vdots \\
0, & 0, & 0, & 0, & \ldots
\end{array}\right)+\left(\begin{array}{ccccc}
1 / 2 !, & -1 / 3 !, & 0, & 0 & \ldots \\
0, & 0, & 0, & 0, & \ldots \\
\vdots & \vdots & \vdots & \ddots & \vdots \\
0, & 0, & 0, & 0, & \ldots
\end{array}\right) \\
& =\left(\begin{array}{ccccc}
1, & 0, & 0, & 0 & \ldots \\
0, & 0, & 0, & 0, & \ldots \\
\vdots & \vdots & \vdots & \ddots & \vdots \\
0, & 0, & 0, & 0, & \ldots
\end{array}\right) \\
d(x+y, x+y)=\sup \left\{\left((m+n) !\left(\left|x_{m n}+y_{m n}\right|-\left|x_{m n}-y_{m n}\right|\right)\right)^{1 / m+n}\right. & : m, n=1,2,3, \ldots\}
\end{aligned}
$$




$$
\begin{aligned}
& d\left(x_{m n}+y_{m n}, 0\right)=\sup \left(\begin{array}{cc}
\left(2 !\left|x_{11}+y_{11}\right|\right)^{1 / 2}, & \left(3 !\left|x_{12}+y_{12}\right|\right)^{1 / 3}, \\
\vdots & \vdots
\end{array}\right) \\
& =\sup \left(\begin{array}{cc}
(2 !|1 / 2 !+1 / 2 !|)^{1 / 2}, & (3 !|1 / 3 !-1 / 3 !|)^{1 / 3}, \\
\vdots & \cdots
\end{array}\right) \\
& =\sup \left(\begin{array}{ccc}
(2)^{1 / 2}, & 0, & \cdots \\
0, & 0, & \cdots \\
\vdots & &
\end{array}\right)=\sup \left(\begin{array}{ccc}
1.414, & 0, & \cdots \\
0, & 0, & \cdots \\
\vdots & \vdots &
\end{array}\right)=1.414
\end{aligned}
$$

Therefore $d(x+y, 0)=1.414$. Similarly $d(x-y, 0)=1.26$

By parellogram law,

$$
\begin{aligned}
{[d(x+y, 0)]^{2}+[d(x-y, 0)]^{2} } & =2\left[(d(x, 0))^{2}+(d(0, y))^{2}\right] \Longrightarrow \\
(1.414)^{2}+1.26^{2} & =2\left[1^{2}+1^{2}\right] \Longrightarrow \\
3.586996 & =4 .
\end{aligned}
$$

Hence it is not satisfied by the law. Therefore $\chi^{2}$ is not an inner product space. Assume that $\chi^{2}$ is a Hilbert space. But then $\chi^{2}$ would satisfy reflexivity condition. [Theorem 4.6.6 [42]] . Proposition 3.5, $\chi^{2}$ is not reflexive. Thus $\chi^{2}$ is not a Hilbert space. This completes the proof.

Proposition $3.7 \chi^{2}$ is rotund.

Proof: Let us take

$$
x=x_{m n}=\left(\begin{array}{ccccc}
1 / 2 !, & 0, & 0, & 0 & \ldots \\
0, & 0, & 0, & 0, & \ldots \\
\vdots & \vdots & \vdots & \vdots &
\end{array}\right) \quad \text { and } \quad y=y_{m n}=\left(\begin{array}{ccccc}
1 / 2 !, & 0, & 0, & 0, & \ldots \\
0, & 0, & 0, & 0, & \ldots \\
\vdots & \vdots & \vdots & \vdots &
\end{array}\right)
$$

Then $x=\left(x_{m n}\right)$ and $y=\left(y_{m n}\right)$ are in $\chi^{2}$. Also

$$
\begin{aligned}
& d(x, y)= \\
& \sup \left(\begin{array}{ccccc}
\left(2 !\left|x_{11}-y_{11}\right|\right)^{\frac{1}{1}}, & \ldots & \left((n+1) !\left|x_{1 n}-y_{1 n}\right|\right)^{\frac{1}{1+n}}, & 0, & \ldots \\
\vdots & \vdots & & & \\
\left((m+1) !\left|x_{m 1}-y_{m 1}\right|\right)^{\frac{1}{m+1}}, & \ldots & \left((m+n) !\left|x_{m n}-y_{m n}\right|\right)^{\frac{1}{m+n}}, & 0, & \ldots \\
0, & \ldots & 0, & \ldots &
\end{array}\right)
\end{aligned}
$$

Therefore

$$
d(x, 0)=\sup \left(\begin{array}{ccccc}
1, & 0, & 0, & 0 & \ldots \\
0, & 0, & 0, & 0, & \cdots \\
\vdots & \vdots & & \vdots & \vdots \\
0, & 0, & 0, & 0, & \ldots
\end{array}\right), \quad d(0, y)=1
$$


Obviously $x=\left(x_{m n}\right) \neq y=\left(y_{m n}\right)$. But

$$
\begin{aligned}
& \left(x_{m n}\right)+\left(y_{m n}\right)=\left(\begin{array}{ccccc}
1 / 2 !, & 0, & 0 & , 0 & \ldots \\
0, & 0, & 0, & 0, & \ldots \\
\vdots & \vdots & \vdots & \vdots &
\end{array}\right)+\left(\begin{array}{ccccc}
1 / 2 !, & 0, & 0, & 0 & \ldots \\
0, & 0, & 0, & 0, & \ldots \\
\vdots & \vdots & \vdots & \vdots &
\end{array}\right) \\
& =\left(\begin{array}{ccccc}
1, & 0, & 0, & 0 & \cdots \\
0, & 0, & 0, & 0, & \cdots \\
\vdots & \vdots & \vdots & \vdots &
\end{array}\right) \\
& d\left(\frac{x_{m n}+y_{m n}}{2}, 0\right) \\
& =\sup \left(\begin{array}{ccccc}
\frac{\left(2 !\left|x_{11}+y_{11}\right|\right)^{1 / 2}}{2}, & \ldots & \frac{\left((1+n) !\left|x_{1 n}+y_{1 n}\right|\right)^{1 / n+1}}{2}, & 0, & \ldots \\
\vdots & \ddots & \vdots & \vdots & \vdots \\
\frac{\left((m+1) !\left|x_{m 1}+y_{m 1}\right|\right)^{1 / m+1}}{2}, & \ldots, & \frac{\left((m+n) !\left|x_{m n}+y_{m n}\right|\right)^{1 / m+n}}{2}, & 0, & \ldots
\end{array}\right) \\
& d\left(\frac{x_{m n}+y_{m n}}{2}, 0\right)=\sup \left(\begin{array}{ccccc}
\left(2^{1 / 2}\right) / 2, & 0, & 0, & 0 & \cdots \\
0, & 0, & 0, & 0, & \cdots \\
\vdots & \vdots & \vdots & \vdots &
\end{array}\right)=0.71
\end{aligned}
$$

Therefore $\chi^{2}$ is rotund. This completes the proof.

Proposition 3.8 Weak convergence and strong convergence are equivalent in $\chi^{2}$.

Proof: Step1: Always strong convergence implies weak convergence.

Step2: So it is enough to show that weakly convergence implies strongly convergence in $\chi^{2} \cdot y^{(\eta)}$ tends to weakly in $\chi^{2}$, where $\left(y_{m n}^{(\eta)}\right)=y^{(\eta)}$ and $y=\left(y_{m n}\right)$. Take any $x=\left(x_{m n}\right) \in \chi^{2}$ and

$$
f(z)=\sum_{m, n=1}^{\infty}\left((m+n) !\left|z_{m n} x_{m n}\right|\right)^{1 / m+n} \text { foreach } z=\left(z_{m n}\right) \in \chi^{2}
$$

Then $f \in\left(\chi^{2}\right)^{*}$ by Proposition 3.2. By hypothesis $f\left(y^{\eta}\right) \rightarrow f(y)$ as $\eta \rightarrow \infty$.

$$
\begin{gathered}
f\left(y^{(\eta)}-y\right) \rightarrow 0 \quad \text { as } \eta \rightarrow \infty . \\
\sum_{m, n=1}^{\infty}\left(\left|y_{m n}^{(\eta)}-y_{m n}\right|^{1 / m+n}((m+n) !)^{1 / m+n}\left|x_{m n}\right|^{1 / m+n}\right) \rightarrow 0 \quad \text { as } \quad \eta \rightarrow \infty
\end{gathered}
$$

By using (6) and (7) we get since $x=\left(x_{m n}\right) \in \Lambda^{2}$ we have

$$
\sum_{m, n=1}^{\infty}\left|x_{m n}\right|^{1 / m+n}<\infty \text { for all } x \in \Lambda^{2} .
$$




$$
\begin{array}{ll}
\Longrightarrow & \sum_{m, n=1}^{\infty}\left((m+n) !\left|y_{m n}^{(\eta)}-y_{m n}\right|\right)^{1 / m+n} \rightarrow 0 \text { as } \eta \rightarrow \infty \\
\Longrightarrow & \sup \left((m+n) !\left|\left(y_{m n}^{(\eta)}-y_{m n}\right), 0\right|\right)^{1 / m+n} \rightarrow 0 \text { as } \eta \rightarrow \infty \\
\Longrightarrow & \sup _{m n}\left((m+n) !\left|y_{m n}^{(\eta)}-y_{m n}\right|\right)^{1 / m+n} \rightarrow 0 \text { as } \eta \rightarrow \infty \\
\Longrightarrow & d\left(\left(y^{(\eta)}-y\right), 0\right) \rightarrow 0 \text { as } \eta \rightarrow \infty . \\
\Longrightarrow & d\left(y^{(\eta)}-y\right) \rightarrow 0 \text { as } \eta \rightarrow \infty .
\end{array}
$$

This completes the proof.

Proposition 3.9 There exists an infinite matrix A for which $\chi_{A}^{2}=\chi^{2}$.

Proof: Consider the matrix

$$
\begin{aligned}
& \left(\begin{array}{ccccccc}
2 ! y_{11}, & 3 ! y_{12}, & \ldots, & (1+n) ! y_{1 n}, & 0, & 0 & \ldots \\
3 ! y_{21}, & 4 ! y_{22}, & \ldots, & (2+n) ! y_{2 n}, & 0, & 0 & \ldots \\
\vdots & \vdots & \vdots & \vdots & \vdots & \vdots & \\
(m+1) ! y_{m 1}, & (m+2) ! y_{m 2}, & \ldots, & (m+n) ! y_{m n}, & 0, & 0 & \ldots \\
0, & 0, & \ldots, & 0, & 0, & 0 & \ldots \\
\vdots & \vdots & \vdots & \vdots & \vdots & \vdots &
\end{array}\right)
\end{aligned}
$$

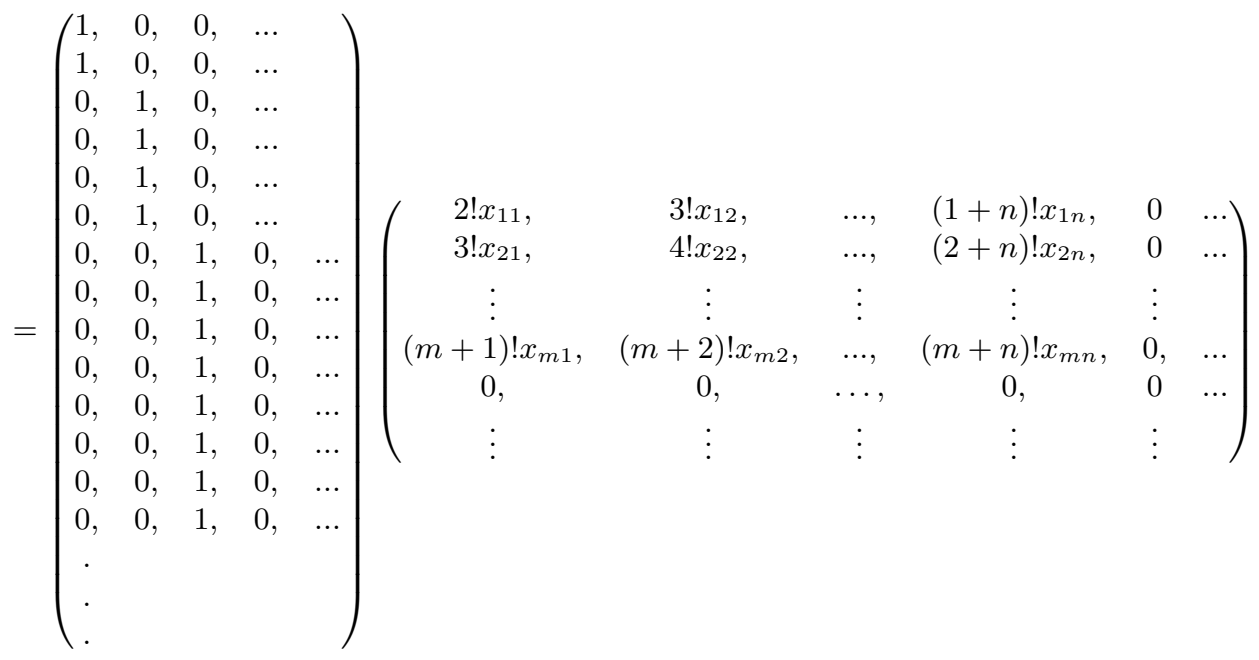




$$
\begin{aligned}
& \left(\begin{array}{ccccccc}
2 ! y_{11}, & 3 ! y_{12}, & \ldots, & (1+n) ! y_{1 n}, & 0, & 0 & \ldots \\
3 ! y_{21}, & 4 ! y_{22}, & \ldots, & (2+n) ! y_{2 n}, & 0, & 0 & \ldots \\
\cdot & & & & & & \\
\cdot & & & & & \\
\cdot & & & & & \\
(m+1) ! y_{m 1}, & (m+2) ! y_{m 2}, & \ldots, & (m+n) ! y_{m n}, & 0, & 0 & \ldots \\
0, & 0, & \ldots, & 0, & 0, & 0 & \ldots \\
\cdot & & & & & & \\
\cdot & & & & & &
\end{array}\right) \\
& =\left(\begin{array}{ccc}
2 ! x_{11}, \ldots, & (1+n) ! x_{1 n}, & 0, \ldots \\
2 ! x_{11}, \ldots, & (1+n) ! x_{1 n}, & 0, \ldots \\
3 ! x_{21}, \ldots, & (2+n) ! x_{2 n}, & 0, \ldots \\
3 ! x_{21}, \ldots, & (2+n) ! x_{2 n}, & 0, \ldots \\
3 ! x_{21}, \ldots, & (2+n) ! x_{2 n}, & 0, \ldots \\
3 ! x_{21}, \ldots, & (2+n) ! x_{2 n}, & 0, \ldots \\
4 ! x_{31}, \ldots, & (3+n) ! x_{3 n}, & 0, \ldots \\
4 ! x_{31}, \ldots, & (3+n) ! x_{3 n}, & 0, \ldots \\
4 ! x_{31}, \ldots, & (3+n) ! x_{3 n}, & 0, \ldots \\
4 ! x_{31}, \ldots, & (3+n) ! x_{3 n}, & 0, \ldots \\
4 ! x_{31}, \ldots, & (3+n) ! x_{3 n}, & 0, \ldots \\
4 ! x_{31}, \ldots, & (3+n) ! x_{3 n}, & 0, \ldots \\
4 ! x_{31}, \ldots, & (3+n) ! x_{3 n}, & 0, \ldots \\
4 ! x_{31}, \ldots, & (3+n) ! x_{3 n}, & 0, \ldots \\
\vdots & \vdots & \vdots
\end{array}\right) \\
& 2 ! y_{11}, \ldots,(1+n) ! y_{1 n}=2 ! x_{11}, \ldots,(1+n) ! x_{1 n} \\
& 3 ! y_{21}, \ldots,(2+n) ! y_{2 n}=2 ! x_{11}, \ldots,(1+n) ! x_{1 n} \\
& 4 ! y_{31}, \ldots,(3+n) ! y_{3 n}=3 ! x_{21}, \ldots,(2+n) ! x_{2 n} \\
& 5 ! y_{41}, \ldots,(4+n) ! y_{4 n}=3 ! x_{21}, \ldots,(2+n) ! x_{2 n} \\
& 6 ! y_{51}, \ldots,(5+n) ! y_{5 n}=3 ! x_{21}, \ldots,(2+n) ! x_{2 n} \\
& 7 ! y_{61}, \ldots,(6+n) ! y_{6 n}=3 ! x_{21}, \ldots,(2+n) ! x_{2 n}
\end{aligned}
$$

and so on. For any $x=\left(x_{m n}\right) \in \chi^{2}$.

$$
\left|(A x)_{m n}\right|=m, n^{\lim } \rightarrow \infty\left((m+n) !\left|\Sigma x_{m n}\right|\right)^{1 / m+n} \leq d(x, 0)
$$

where metric is taken $\chi^{2}$.

$$
[d(x, 0)]_{\chi_{A}^{2}} \leq[d(x, 0)]_{\chi^{2}}
$$


Conversely, Given $x \in[d(x, 0)]_{\chi_{A}^{2}}$ fix any $m, n$ then,

$$
\begin{aligned}
m, n \rightarrow \infty & \lim \\
\Longrightarrow \quad m\left((m+n) !\left|x_{m n}\right|\right)^{1 / m+n} & \leq(A x)_{m n} . \\
\lim _{\rightarrow} \infty\left((m+n) !\left|x_{m n}\right|\right)^{1 / m+n} & \leq[d(x, 0)]_{\chi_{A}^{2}} \\
{[d(x, 0)]_{\chi^{2}} } & \leq[d(x, 0)]_{\chi_{A}^{2}} .
\end{aligned}
$$

Therefore the matrix $A=\left(x_{m n}^{\ell k}\right)$ for whcich the summability field $[d(x, 0)]_{\chi^{2}}=[d(x, 0)]_{\chi_{A}^{2}}$ is given by

$$
A=\left(\begin{array}{ccccc}
1, & 0, & 0, & \ldots & \\
1, & 0, & 0, & \ldots & \\
0, & 1, & 0, & \ldots & \\
0, & 1, & 0, & \ldots & \\
0, & 1, & 0, & \ldots & \\
0, & 1, & 0, & \ldots & \\
0, & 0, & 1, & 0, & \ldots \\
0, & 0, & 1, & 0, & \ldots \\
0, & 0, & 1, & 0, & \ldots \\
0, & 0, & 1, & 0, & \ldots \\
0, & 0, & 1, & 0, & \ldots \\
0, & 0, & 1, & 0, & \ldots \\
0, & 0, & 1, & 0, & \ldots \\
0, & 0, & 1, & 0, & \ldots \\
\vdots & & & &
\end{array}\right)
$$

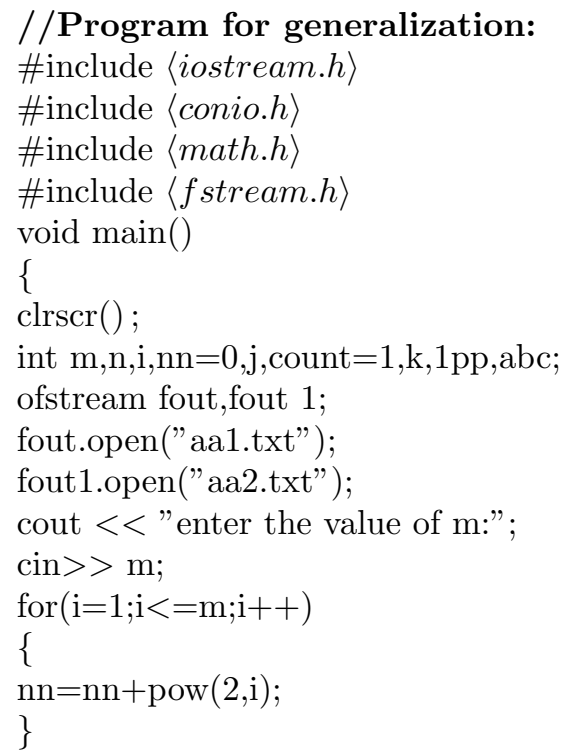


$\mathrm{i}=0$

while $($ count $<=$ nn $)$

\{

cout $<<"-"$;

fout $<<"-"$;

for $(\mathrm{abc}=1 ; \mathrm{abc}<=\mathrm{m}+2 ; \mathrm{abc}++)$ 


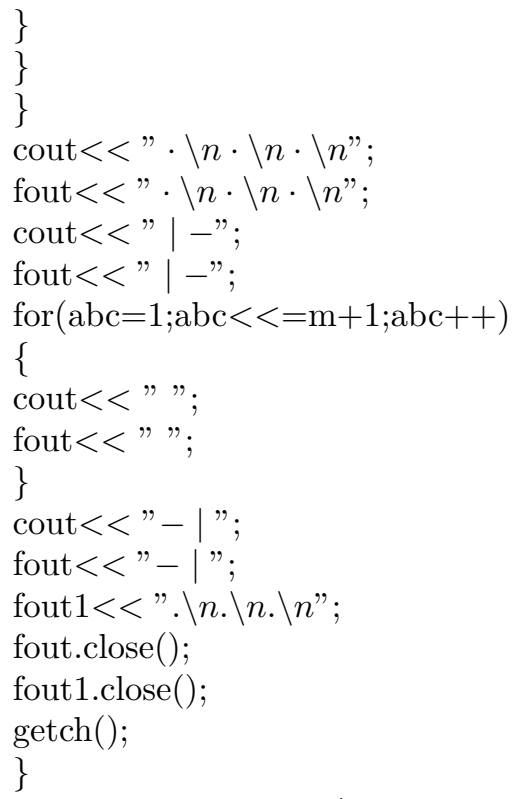

SAMPLE INPUT/OUTPUT:

Enter the value of $\mathrm{m}=3$

$$
\begin{aligned}
& \left(\begin{array}{lllll}
1, & 0, & 0, & \ldots & \\
1, & 0, & 0, & \ldots & \\
0, & 1, & 0, & \ldots & \\
0, & 1, & 0, & \ldots & \\
0, & 1, & 0, & \ldots & \\
0, & 1, & 0, & \ldots & \\
0, & 0, & 1, & 0, & \ldots \\
0, & 0, & 1, & 0, & \ldots \\
0, & 0, & 1, & 0, & \ldots \\
0, & 0, & 1, & 0, & \ldots \\
0, & 0, & 1, & 0, & \ldots \\
0, & 0, & 1, & 0, & \ldots \\
0, & 0, & 1, & 0, & \ldots \\
0, & 0, & 1, & 0, & \ldots \\
\vdots & & & &
\end{array}\right) \\
& 2 ! Y_{1,1}, \ldots,(1+n) ! Y_{1, n}=2 ! X_{1,1}, \ldots,(1+n) ! X_{1, n} \\
& 3 ! Y_{2,1}, \ldots,(2+n) ! Y_{2, n}=2 ! X_{1,1}, \ldots,(1+n) ! X_{1, n} \\
& 4 ! Y_{3,1}, \ldots,(3+n) ! Y_{3, n}=3 ! X_{2,1}, \ldots,(2+n) ! X_{2, n} \\
& 5 ! Y_{4,1}, \ldots,(4+n) ! Y_{4, n}=3 ! X_{2,1}, \ldots,(2+n) ! X_{2, n} \\
& 6 ! Y_{5,1}, \ldots,(5+n) ! Y_{5, n}=3 ! X_{2,1}, \ldots,(2+n) ! X_{2, n} \\
& 7 ! Y_{6,1}, \ldots,(6+n) ! Y_{6, n}=3 ! X_{2,1}, \ldots,(2+n) ! X_{2, n}
\end{aligned}
$$




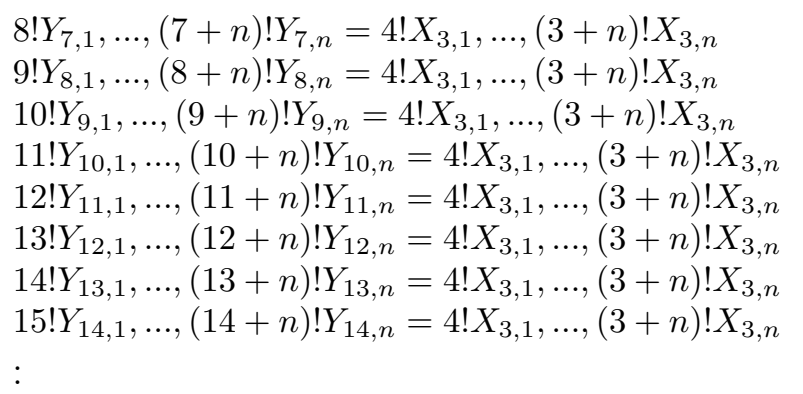

Acknowledgement: I wish to thank the referee's for their several remarks and valuable suggestions that improved the presentation of the paper.

\section{References}

[1] T.Apostol, Mathematical Analysis, Addison-wesley, London, 1978.

[2] M.Basarir and O.Solancan, On some double sequence spaces, J. Indian Acad. Math., 21(2) (1999), 193-200.

[3] C.Bektas and Y.Altin, The sequence space $\ell_{M}(p, q, s)$ on seminormed spaces, Indian J. Pure Appl. Math., 34(4) (2003), 529-534.

[4] T.J.I'A.Bromwich, An introduction to the theory of infinite series Macmillan and Co.Ltd. ,New York, (1965).

[5] J.C.Burkill and H.Burkill, A Second Course in Mathematical Analysis Cambridge University Press, Cambridge, New York, (1980).

[6] R.Colak and A.Turkmenoglu, The double sequence spaces $\ell_{\infty}^{2}(p), c_{0}^{2}(p)$ and $c^{2}(p)$, (to appear).

[7] M.Gupta and P.K.Kamthan,Infinite matrices and tensorial transformations, Acta Math., Vietnam 5 (1980), 33-42.

[8] G.H.Hardy, On the convergence of certain multiple series, Proc. Camb. Phil. Soc., 19 (1917), 86-95.

[9] M.A.Krasnoselskii and Y.B.Rutickii, Convex functions and Orlicz spaces, Gorningen, Netherlands, 1961.

[10] J.Lindenstrauss and L.Tzafriri, On Orlicz sequence spaces, Israel J. Math., 10 (1971), 379-390.

[11] I.J.Maddox, Sequence spaces defined by a modulus, Math. Proc. Cambridge Philos. Soc, 100(1) (1986), 161-166.

[12] F.Moricz, Extentions of the spaces $c$ and $c_{0}$ from single to double sequences, Acta. Math. Hungerica, 57(1-2), (1991), 129-136. 
[13] F.Moricz and B.E.Rhoades, Almost convergence of double sequences and strong regularity of summability matrices, Math. Proc. Camb. Phil. Soc., 104, (1988), 283-294.

[14] M.Mursaleen,M.A.Khan and Qamaruddin, Difference sequence spaces defined by Orlicz functions, Demonstratio Math. , Vol. XXXII (1999), 145-150.

[15] H.Nakano, Concave modulars, J. Math. Soc. Japan, 5(1953), 29-49.

[16] W.Orlicz, Über Raume $\left(L^{M}\right)$ Bull. Int. Acad. Polon. Sci. A, (1936), 93-107.

[17] S.D.Parashar and B.Choudhary, Sequence spaces defined by Orlicz functions, Indian J. Pure Appl. Math. , 25(4)(1994), 419-428.

[18] K.Chandrasekhara Rao and N.Subramanian, The Orlicz space of entire sequences, Int. J. Math. Math. Sci., 68(2004), 3755-3764.

[19] W.H.Ruckle, FK spaces in which the sequence of coordinate vectors is bounded, Canad. J. Math., 25(1973), 973-978.

[20] B.C.Tripathy, On statistically convergent double sequences, Tamkang J. Math., 34(3), (2003), 231-237.

[21] B.C.Tripathy,M.Et and Y.Altin, Generalized difference sequence spaces defined by Orlicz function in a locally convex space, J. Analysis and Applications, 1(3)(2003), 175-192.

[22] A.Turkmenoglu, Matrix transformation between some classes of double sequences, Jour. Inst. of math. and Comp. Sci. (Math. Seri. ), 12(1), (1999), 23-31.

[23] A.Wilansky, Summability through Functional Analysis, North-Holland Mathematics Studies, North-Holland Publishing, Amsterdam, Vol.85(1984).

[24] P.K.Kamthan and M.Gupta, Sequence spaces and series, Lecture notes, Pure and Applied Mathematics, 65 Marcel Dekker, In c., New York , 1981.

[25] M.Gupta and P.K.Kamthan, Infinite Matrices and tensorial transformations, Acta Math. Vietnam 5, (1980), 33-42.

[26] N.Subramanian, R.Nallswamy and N.Saivaraju, Characterization of entire sequences via double Orlicz space, Internaional Journal of Mathematics and Mathemaical Sciences, Vol.2007(2007), Article ID 59681, 10 pages.

[27] A.Gökhan and R.Colak, The double sequence spaces $c_{2}^{P}(p)$ and $c_{2}^{P B}(p), A p p l$. Math. Comput., 157(2), (2004), 491-501.

[28] A.Gökhan and R.Colak, Double sequence spaces $\ell_{2}^{\infty}$, ibid., 160(1), (2005), $147-$ 153. 
[29] M.Zeltser, Investigation of Double Sequence Spaces by Soft and Hard Analitical Methods, Dissertationes Mathematicae Universitatis Tartuensis 25, Tartu University Press, Univ. of Tartu, Faculty of Mathematics and Computer Science, Tartu, 2001.

[30] M.Mursaleen and O.H.H. Edely, Statistical convergence of double sequences, $J$. Math. Anal. Appl., 288(1), (2003), 223-231.

[31] M.Mursaleen, Almost strongly regular matrices and a core theorem for double sequences, J. Math. Anal. Appl., 293(2), (2004), 523-531.

[32] M.Mursaleen and O.H.H. Edely,Almost convergence and a core theorem for double sequences, J. Math. Anal. Appl., 293(2), (2004), 532-540.

[33] B.Altay and F.Basar, Some new spaces of double sequences, J. Math. Anal. Appl., 309(1), (2005), 70-90.

[34] F.Basar and Y.Sever, The space $\mathcal{L}_{p}$ of double sequences, Math. J. Okayama Univ, 51, (2009), 149-157.

[35] N.Subramanian and U.K.Misra, The semi normed space defined by a double gai sequence of modulus function, Fasciculi Math., 46, (2010).

[36] H.Kizmaz, On certain sequence spaces, Cand. Math. Bull., 24(2), (1981), 169176.

[37] N.Subramanian and U.K.Misra, Characterization of gai sequences via double Orlicz space, Southeast Asian Bulletin of Mathematics, (revised).

[38] N.Subramanian, B.C.Tripathy and C.Murugesan, The double sequence space of $\Gamma^{2}$, Fasciculi Math. , 40, (2008), 91-103.

[39] N.Subramanian, B.C.Tripathy and C.Murugesan, The Cesáro of double entire sequences, International Mathematical Forum, 4 no.2(2009), 49-59.

[40] N.Subramanian and U.K.Misra, The Generalized double of gai sequence spaces, Fasciculi Math., 43, (2010).

[41] N.Subramanian and U.K.Misra, Tensorial transformations of double gai sequence spaces, International Journal of Computational and Mathematical Sciences, 3:4, (2009), 186-188.

[42] Erwin Kreyszig, Introductory Functional Analysis with Applications, by John wiley and sons Inc. , 1978.

[43] M.Mursaleen and S.A.Mohiuddine, Regularly $\sigma-$ conservative and $\sigma-$ coercive four dimensional matrices, Computers and Mathematics with Applications, 56(2008), 1580-1586. 
[44] M.Mursaleen and S.A.Mohiuddine, On $\sigma-$ conservative and boundedly $\sigma-$ conservative four dimensional matrices, Computers and Mathematics with Applications, 59(2010), 880-885.

[45] M.Mursaleen and S.A.Mohiuddine, Double $\sigma-$ multiplicative matrices, J. Math. Anal. Appl. , 327(2007), 991-996.

DOI: $10.7862 / \mathrm{rf.2013.9}$

N.Subramanian - corresponding author

email: nsmaths@yahoo.com

Department of Mathematics,SASTRA University,

Thanjavur-613 401, India.

\section{U.K.Misra}

email: umakanta_misra@yahoo.com

Department of Mathematics, Berhampur University,

Berhampur-760 007, Odissa, India

Received 05.03.2013, Revisted 10.05.2013, Accepted 25.10.2013 